\title{
Journal of Neurological Disorders
}

\section{Support at Home: To Enhance Life in Dementia}

\section{Chao-Jun Kong ${ }^{1}$, Jie Zhang ${ }^{2}$ and Zhong Jia ${ }^{2 *}$}

${ }^{1}$ Zhejiang Chinese Medical University, Zhejiang Chinese Medical Affiliated Fouth Clinical Medical Hospital, P.R. China

${ }^{2}$ Hangzhou First People's Hospital, Nanjing Medical University Affiliated Hangzhou Hospital, Huansha Road Hangzhou, P.R. China

\section{Description}

Dementia, including Alzheimer's disease, is becoming a national prioritized concern due to the rapid increase in prevalence, as well as the potential social burden [1]. Importantly, an effective drug will not be available to treat Alzheimer's disease until 2025 [2]. Thus, family members' joint efforts play a realistic role for improvement of patients with dementia.

Indeed, kinship with emotional intelligence will improve mental diseases like dementia. Based on authors' opinion, these practical skills at home may be helpful. First and foremost, the personality traits of family caregivers may influence the outcomes of patients with dementia [3]. Family members should be patient and take time to listen to what older seniors with dementia say. The wise response is to support them with a positive outlook. Any intended or undesired micro-discrimination showing the appearance of members' faces will unexpectedly harm older seniors with dementia. Aging seniors with dementia are somewhat fragile and sensitive, partly due to brain atrophy [4]. Second, touch can be used as an incentive to transmit trust or inspiration. Specifically, family members can give the patient a close embrace or walk together, which evoke deep memory. Physical touching is also a basic element of social behavior that prevents social isolation or self-isolation. Third, dementia is characterized by forgetfulness, especially shortterm memory, such as forgetting recent events or confusing familiar surroundings or people. Those affected with dementia are unaware that their abilities have deteriorated. As a result, they often argue about what is right or wrong with a stubborn attitude.

All family members should keep a peaceful and inclusive mindset to produce or create an environment to allow mutual self-adjustment. Then, having learned that everything has two sides (like a coin), it is important to let issues go, and internalize emotional responses. In fact, quarrelling also represents a type of "biologic therapy." Quarrelling can be beneficial by continuously stimulating mental activities, as well as releasing bad emotions; however, family members must keep their emotions in check. Generally, caregivers should not take sides, but be ready to step in when the situation deteriorates. Dilemma-solving skills (i.e., emotional intelligence) are needed to get along well with each other. More importantly, the patient with dementia needs to enjoy family time at regular interval rather than special days alone, which means family ties, such as, having meals together. Indeed, family activities serve as a substantial palliative drug, targeting to live a simple, but functional life with value-based dignity. In these ways, kinship-oriented support is powerful.

\section{References}

1. Amjad H, Carmichael D, Austin AM, Chang CH, Bynum JPW (2016) Continuity of care and health care utilization in older adults with dementia in fee-forservice Medicare. JAMA Intern Med 176: 1371-1378.

2. Orrell M, Hoe J, Charlesworth G, Russell I, Challis D, et al. (2017) Support at Home: Interventions to Enhance Life in Dementia (SHIELD) - Evidence, development and evaluation of complex interventions. NHR Journals Library. Southampton, UK. 5: 1-184.

3. Merrilees $J$ (2016) The impact of dementia on family caregivers: What is research teaching us? Curr Neurol Neurosci Rep 16: 88.

4. Nho K, Saykin AJ, Nelson PT (2016) Hippocampal sclerosis of aging, a common alzheimer's disease 'mimic': Risk genotypes are associated with brain atrophy outside the temporal lobe. J Alzheimer's Dis 52: 373-383.

*Corresponding author: Zhong Jia, Hangzhou First People's Hospital, Nanjing Medical University Affiliated Hangzhou Hospital, Huansha Road 261, Hangzhou 310006, P.R. China, Tel: 86-13958114181; Fax: 86-0571- 87914773; E-mail: jiazhong20058@hotmail.com

Received July 22, 2017; Accepted July 31, 2017; Published August 02, 2017

Citation: Kong C, Zhang J, Jia Z (2017) Support at Home: To Enhance Life in Dementia. J Neurol Disord 5: 355. doi:10.4172/2329-6895.1000355

Copyright: $\odot 2017$ Kong C, et al. This is an open-access article distributed under the terms of the Creative Commons Attribution License, which permits unrestricted use, distribution, and reproduction in any medium, provided the original author and source are credited. 